\title{
Finite Element Analysis of Adhesive Steel Bar in Concrete under Tensile Load
}

\author{
Jianrong Zhang ${ }^{1, a}$, Ruibin Shu ${ }^{2, b}$ and Zixiang Zhao ${ }^{3, c}$ \\ 1,2,3 Tongji University, Shanghai, 200092, China

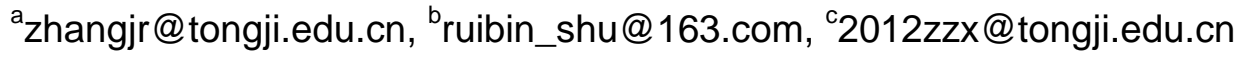

\begin{abstract}
Keywords: adhesive anchorage technology; bond-slip relationship; finite element analysis; stress distribution; crack development

Abstract. According to the results of pullout-test for adhesive steel bar in concrete, a bond-slip relationship on adhesive interface is established. This relationship reflects effect of some parameters, such as effective embedment length, drill diameter, characters of anchor adhesive, etc., on bonding strength and slip of mixed interface. The finite element method was used for the analysis of adhesive anchorage system. The pull out loading process was simulated and a complete tension-displacement curve of the steel bar was obtained by finite element analysis. The distribution of stress and the development of cracks in concrete can be described in detail according to numerical computation. The analytical results coincide with the experimental results which proved that the analysis model is effective and the parameters are reliable. Through a large number of numerical calculations on adhesive anchorage system, the basic laws of stress distribution in steel bar, adhesive glue and concrete are obtained for different embedment length. The model provides the analytical tools and basic method for the analysis of adhesive anchorage system in micro-level.
\end{abstract}

\section{Introduction}

As a post-installed fixing technology, adhesive anchor is widely used in civil engineering for installation of nonstructural component and building decoration. Meanwhile, with the same technique, adhesive steel bar is also widely used in engineering reconstruction or structural rebuilding. Test results shown that the failure models of adhesive steel bar in concrete are similar as those of adhesive anchors ${ }^{[1]}$. When the embedment depth is small, the concrete core would lead to failure. And when the embedment length is longer than the critical length, the steel bar would lead to failure. In addition to above two failure models, when the embedment depth is less than critical length, failure would happen on mixed interfaces of adhesive/concrete interface and adhesive/steel interface. It seems that the failure model can be generally controlled by the design of steel embedment depth, but in fact, the failure mechanism of adhesive steel in concrete system is comprehensive. When the adhesive steel bar is used as reinforcement in concrete structure, the stress distribution and crack development in concrete structural element would be complex. Because of the limitations of experimental technology, many factors influencing bonding strength and steel deformation cannot be observed or measured in microscopic level. Finite element analysis is an effective method for the study of interaction between adhesive, concrete and steel. By the result of experimental research and finite element analysis, the bonding behavior of adhesive steel bar in concrete can be describe in detail and in micro-level, with the loading process to failure. Based on the nonlinear finite element analysis method, bonding deformation of steel bar under tensile force, stress distribution and development of crack for adhesive steel bar in concrete are obtained. The research provides analytical model in micro-level as the basis of research.

\section{Bond-slip Relationship}

Bond-slip relationship is the basic condition for the finite element analysis of adhesive steel bar in concrete. The results of pull out tests ${ }^{[1,2]}$ were used to establish bond-slip relationship. Reference [1] reported the pullout test of 18 adhesive steel specimens. The specimens were grouped according to 
the embedment length, which were $8 \mathrm{~d}, 10 \mathrm{~d}, 12 \mathrm{~d}$ and $15 \mathrm{~d}$, where $\mathrm{d}$ was the diameter of steel bar. The steel prepared for specimen was a common product as those used in building construction. The adhesive material was the product of Fischer (Taicang) Fixing Co. Ltd., FISV360S, which is a special product for embedment of reinforcement in concrete engineering. The experiment was designed to pull the steel bar to failure under tensile load. Through observing the experiment process, measuring the critical loads and analyzing the failure mechanism, the behavior of adhesive reinforcement under tensile load was studied and described. Reference [2] reported other 3 adhesive steel bar specimens in accordance with three effective embedment length (8d, 10d and 12d). FISV360S was also used for the preparation of the specimen. Through observing the experiment process, measuring the displacement and strain of steel bars, the behavior of adhesive steel bar in concrete was analyzed. Some problems in adhesive steel bar were discussed, such as the failure process, the failure model, the bond mechanism and the distribution of shear stress. The results show that when the effective embedment length is longer than $15 \mathrm{~d}$, steel failure would happen. And when the effect embedment length is $12 \mathrm{~d}$ or less, adhesive interface failure would happen. Results from the test mentioned above shown that the pullout process of the steel bar under tensile load could be simplified as shown in Fig.1.

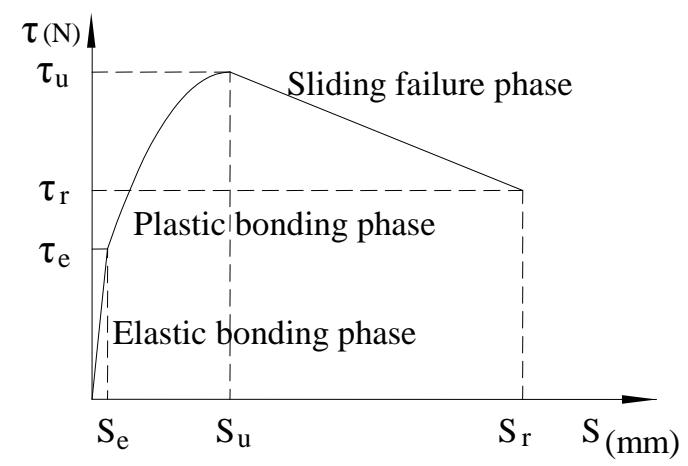

Fig.1 Bond-slid relationship

In Fig.1, $\tau$ means the average shear stress related to the pull force. $s$ means the displacement of the steel bar. Based on uniform bond stress model ${ }^{[3]}$, the average shear stress $\tau$ can be calculated by the pull force $N$ :

$$
\tau=\frac{N}{\pi D h_{e f}}
$$

Where, $D$ is drill diameter $(\mathrm{mm}) ; h_{e f}$ is effective embedment length of steel bar $(\mathrm{mm})$.

From the form of the curve in Fig.1, the whole bond-slip process can be divided into three phases, which are elastic bonding phase, plastic bonding phase and sliding failure phase. In the elastic bonding phase, the curve could be simplified as a ramp straight through the origin. The critical point at the end of elastic bonding phase is $\left(\tau_{e}, s_{e}\right)$. Curve of plastic bonding phase could be simplified as a parabola which vertex strands achieves bonding strength $\left(\tau_{u}, s_{u}\right)$. And the curve of sliding failure phase could be simplified as a decreasing ramp straight with the end point $\left(\tau_{r}, s_{r}\right)$. By the statistic of experimental results ${ }^{[1,2]}$, the bond-slip relationship can be expressed as Equation (2).

$$
\tau=\left\{\begin{array}{lr}
2.68 \frac{\tau_{u}}{S_{u}} \cdot S & \left(0 \leq S<S_{e}\right) \\
-0.48 \frac{\tau_{u}}{S_{u}^{2}} \cdot S^{2}+0.96 \frac{\tau_{u}}{S_{u}} \cdot S+0.52 \tau_{u} & \left(S_{e} \leq S<S_{u}\right) \\
\frac{0.50}{S_{u}-5} \tau_{u} \cdot S+\frac{0.75 S_{u}-5}{S_{u}-5} \tau_{u} & \left(S_{u} \leq S \leq S_{r}\right)
\end{array} .\right.
$$


The Chinese industry standard, Technical specification for post-installed fastenings in concrete structures $^{[4]}$, regresses the design formula based on a vast test results of tension-resistance bearing capacity $\left(N_{u, p a}\right)$, which is suitable for the pullout failure on adhesive-concrete interface:

$$
N_{u, p a}=5.6 h_{e f} D \sqrt{f_{c u}} \text {. }
$$

Where, $f_{c u}$ is cube compressive strength of concrete (MPa).

In this way, the design formula of ultimate bond strength $\tau_{u}$ is:

$$
\tau_{u}=N_{u} /\left(\pi D h_{e f}\right)=5.6 \alpha / \pi \sqrt{f_{c u}} .
$$

\section{Finite Element Analysis Model}

Finite element analysis based on ANSYS program was conducted for the analysis of chemical anchorage system. The ANSYS model diagram, with a quarter of specimen, is showed in Fig.2. In order to consider the interaction on the bonding interface comprehensively, three nonlinear spring elements (Combin39 element) are arranged on the corresponding nodes to simulate the bond-slip relationship between adhesive and concrete. The nodes are between the interface of adhesive and concrete. Three series of springs represent normal interaction, longitudinal tangential interaction and horizontal tangential interaction respectively on the bonding interface. The length of the spring is simulated near zero and the performance of each spring is determined by the force-deformation curve (F-D curve). The bond-slip performance of adhesive-concrete interface is presented by longitudinal tangential spring, which F-D curve is confirmed by the bond-slip relationship on adhesive-concrete interface (Equation 2). Considering iterative solving process of various spring element lead to long computation time and poor astringency, the same F-D curve was adopted for different longitudinal tangential spring elements. On the other hand, the adoption of simple uniform bond stress model can obtain an acceptable result.

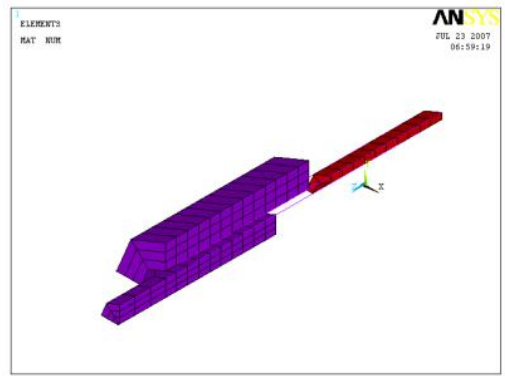

(a) adhesive separation

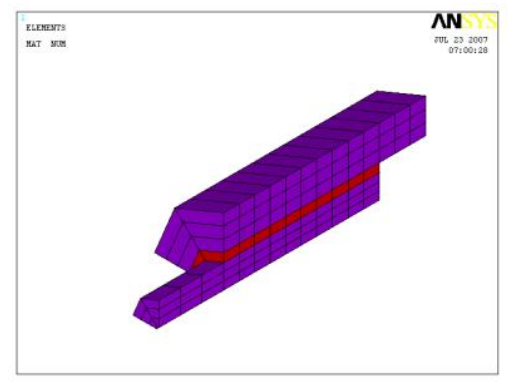

(b) adhesive restoration

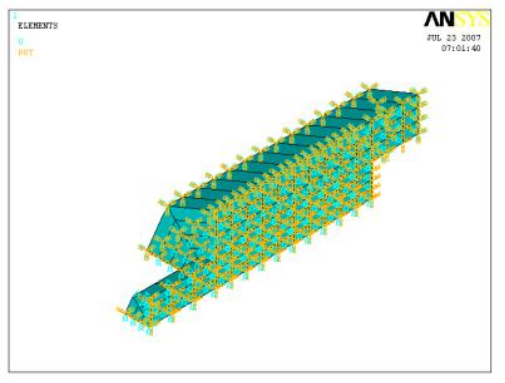

(c) constraint imposition

Fig.2 Finite Element Analysis Model

The F-D curve mentioned above could be derived from the bond-slip relationship on mixed interface for adhesive steel bar. The bond-slip relationship can be obtained by the experiment result of load-displacement curve throughout whole loading process. According to the regression analysis of test results, the relationship of bonding stress and slip can be defined as three segmental phases. As to adhesive brand FISV360S, by statistical analysis of test results ${ }^{[1,2]}$, some indexes at the critical points $\left(\tau_{e}, s_{e}\right),\left(\tau_{u}, s_{u}\right),\left(\tau_{r}, s_{r}\right)$ can be calculated out, $\tau_{u}=6.790 \mathrm{Mpa} ; \tau_{\mathrm{e}}=0.75 \tau_{u} ; \tau_{\mathrm{r}}=0.5 \tau_{u}$; $S_{\mathrm{u}}=1.26 \mathrm{~mm} ; S_{\mathrm{e}}=0.35 \mathrm{~mm} ; S_{\mathrm{r}}=5.00 \mathrm{~mm}$. Replace the above data into Equation (3), the bond-slip relationship for the analysis of the specimen is: 


$$
\tau(s)=\left\{\begin{array}{lr}
14.44 s & 0<s \leq 0.350 \\
-2.05 s^{2}+5.18 s+3.53 & 0.350<s \leq 1.260 \\
-0.91 s+7.94 & 1.260<s \leq 5.000
\end{array} .\right.
$$

With the finite element model and the bond-slip relation, the loading process can be simulated by computation in various forms of result. Figure 3 shows the result of load-displacement relationship. Both the experimental results and finite element analysis results were shown in one diagram for comparison. With the failure model of rebar poll-out, the displacement values of specimens with different anchoring length under different load levels are shown in Figure 3 (a) (b) (c). It can be seen that the calculating results are quite coincide with the experimental results. With the steel failure model as is shown in Figure 3 (d), things is a little bit different. Before steel yields, the steel displacement is mainly provided by the slip on adhesive-concrete interface and the calculating results are quite close to the experimental results. When the steel yields, the bonding force unloading rapidly, which leads to decreasing of slip on adhesive interface.

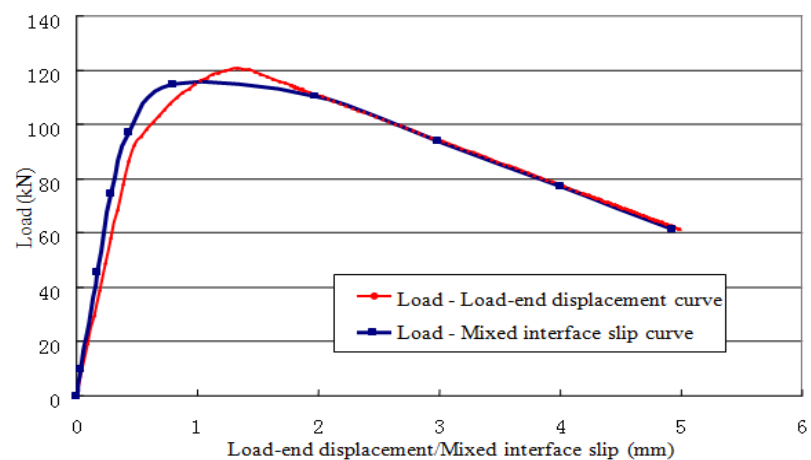

(a) $h_{\mathrm{ef}}=8 \mathrm{~d}$

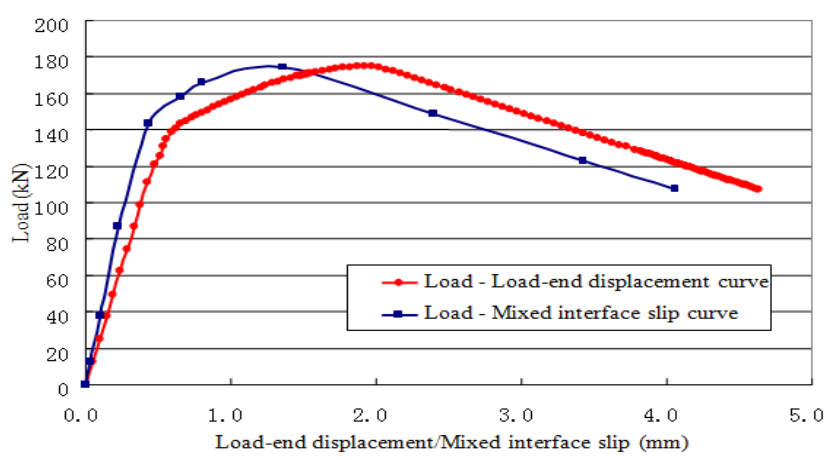

(c) $h_{\mathrm{ef}}=12 \mathrm{~d}$

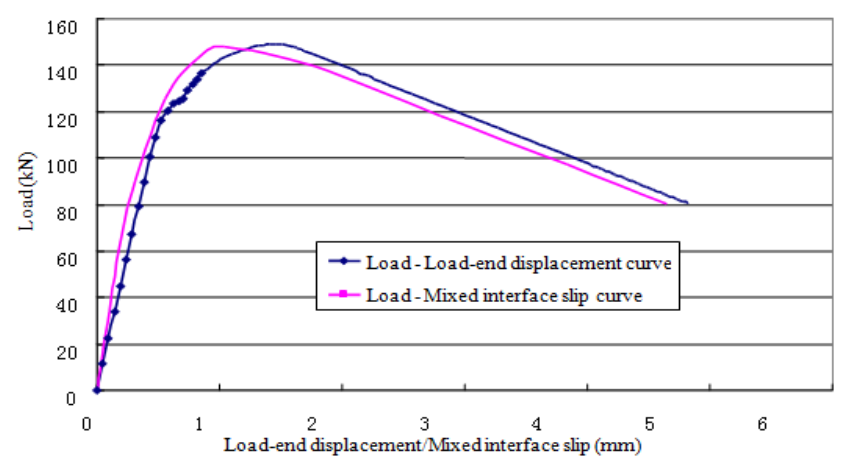

(b) $h_{e f}=10 d$

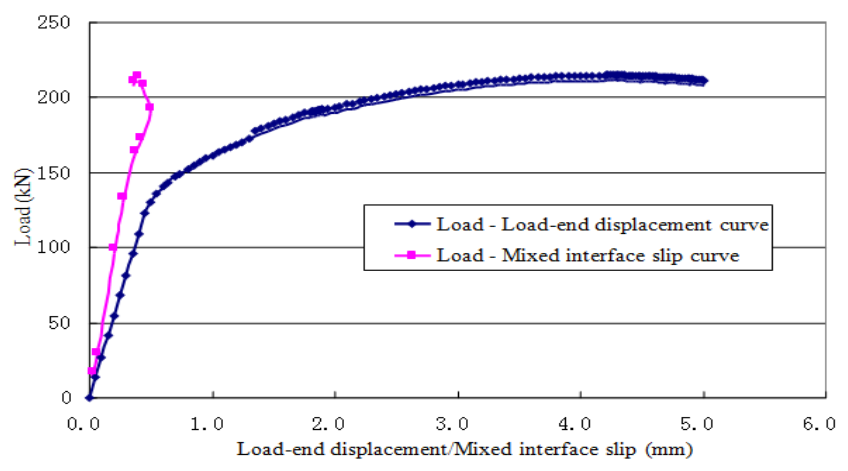

(d) $h_{\text {ef }}=15 d$

Fig.3 Comparison of load-slip curve

\section{Behavior of Adhesive Anchorage System}

Bonding Stress Distribution. With the finite element analysis model as discussed above, the behavior of adhesive steel bar in concrete can be analyzed in detail. The bonding stress distribution for the specimens with different embedment length under different load levels are shown in Figure 4, in which the effective embedment lengths are $8 \mathrm{~d}, 10 \mathrm{~d}, 12 \mathrm{~d}, 15 \mathrm{~d}$, in accordance to (a) (b) (c) (d). The different curves mean different slid displacement related to different level of loading. The result represents that in the initial phase of loading, the bonding stress distribution is uneven and the maximum stress appeared near the concrete surface of loading. In later phase of loading, the stress distribution along the embedment depth tends to an even distribution. In addition to a stress increasing phase on loading side and a stress decreasing phase on the end of rebar, the bonding stress seems even distribution along the rest middle part of the bar for specimens of Fig. 4(a)(b)(c), for mixed interface failure model. The interfacial bond-stress distribution of Fig. 4(d) is different from 
other three, as the specimen failed in steel yield. The stress decreases along the embedment depth of the steel, not in even distribution form.

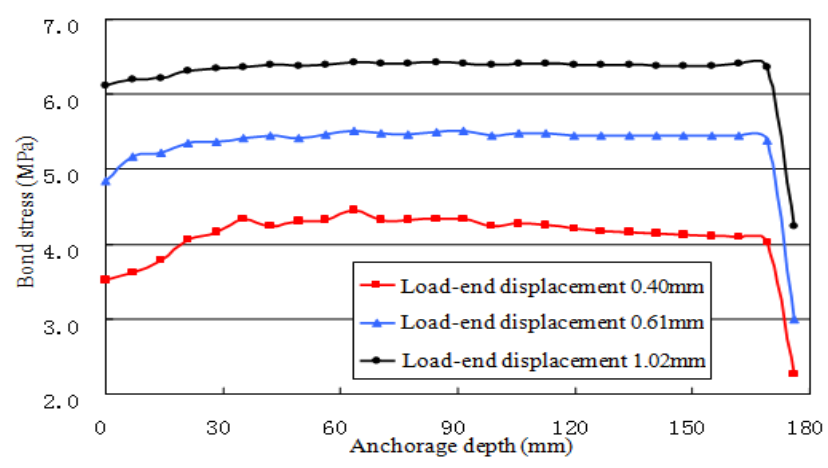

(a) $h_{e f}=8 d$

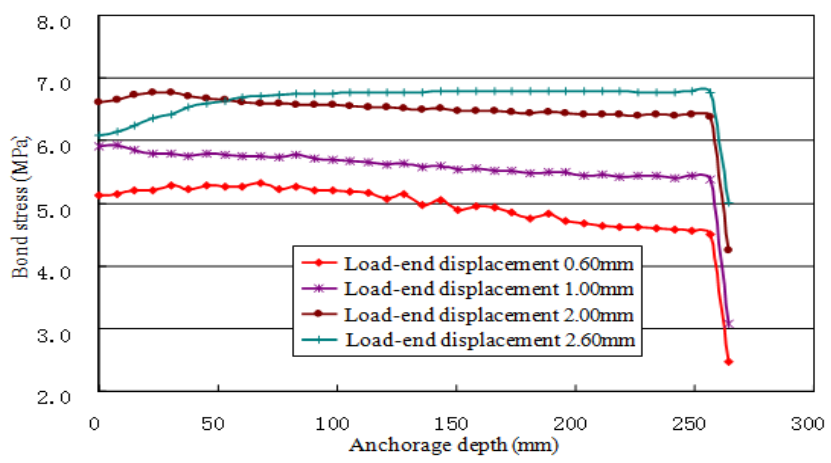

(c) $h_{\mathrm{ef}}=12 \mathrm{~d}$

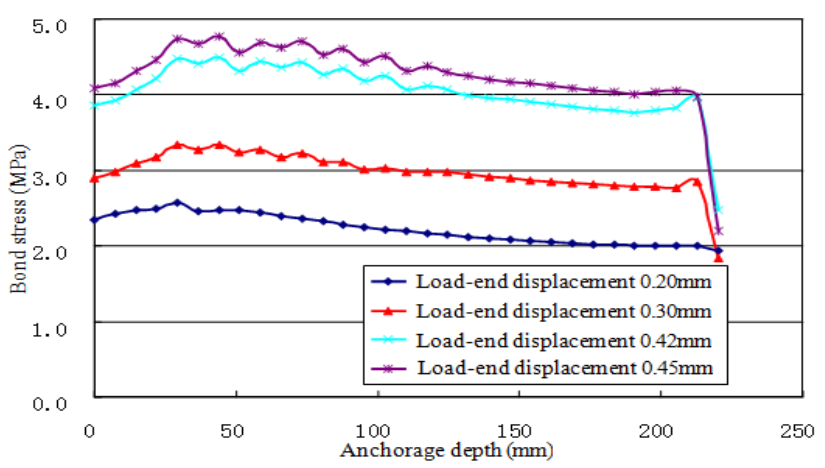

(b) $h_{\mathrm{ef}}=10 \mathrm{~d}$

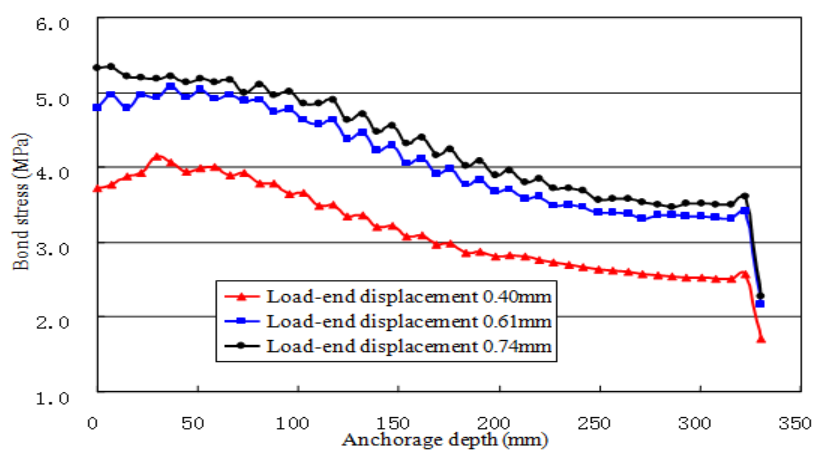

(d) $h_{\text {ef }}=15 d$

Fig.4 Bond-stress distribution on adhesive-concrete interface

Crack Distribution and Development. To reveal the distribution and development of crack on adhesive anchorage system, one of the specimens with effective embedment length of $8 \mathrm{~d}$, was analyzed with finite element method. As is shown in Figure 5, 4 levels of tensile force is expressed as displacement of rebar, $0.005 \mathrm{~mm}, 0.26 \mathrm{~mm}, 0.37 \mathrm{~mm}, 1.02 \mathrm{~mm}$. When the tensile force is small, as is shown in Fig.5 (a), the tensile stress in concrete is in even distribution along the anchorage height of rebar. The adhesive layer is also in tension along the steel rebar. With the increase of the tensile force, Figure 5 (b) and (c) shows the development of stress distribution and crack in concrete. The slant cracks appeared first at adhesive-concrete interface where peak bond stress existed. Slant cracks extended to concrete surface and formed concrete inverted cone. In the wake of interfacial peak bond stress, cracks extended gradually to interior along the embedment rebar. The concrete inverted cone formed continuously with the development of cracks along the embedment steel bar. Figure 5 (d) shows the stress distribution at the later phase of loading. Several rounds of inverted cone type cracks formed along the embedment depth. Because of lack of constraint by concrete at the surface of the specimen, the shallow inverted concrete cone would be formed earlier than the deeper cones. The concrete cone leaves away from the concrete body as the steel was pulled out with sliding.

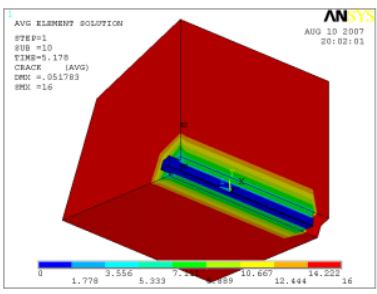

(a) $\mathrm{s}=0.005 \mathrm{~mm}$

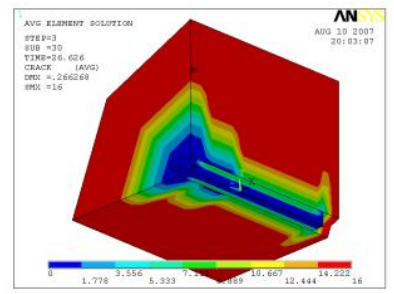

(b) $\mathrm{s}=0.26 \mathrm{~mm}$

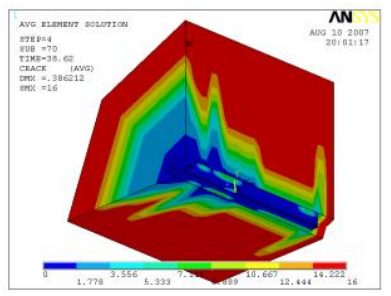

(c) $\mathrm{s}=0.37 \mathrm{~mm}$

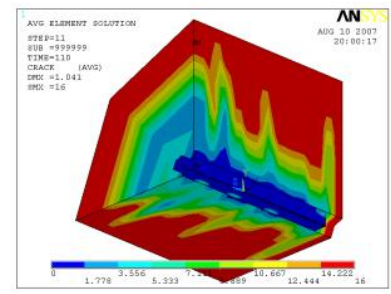

(d) $\mathrm{s}=1.02 \mathrm{~mm}$

Fig.5 Crack development in concrete 
Distribution of Longitudinal Normal Stress. Figure 6 is a longitudinal stress diagram on adhesive steel bar system when embedment length is $8 \mathrm{~d}$. The tensile stress of rebar is decreased with the increase of embedment length. Majority of anchor adhesive is compressed and top cone part of it is in tension. Majority of concrete substrate is compressed and top cone part of it is in tension.

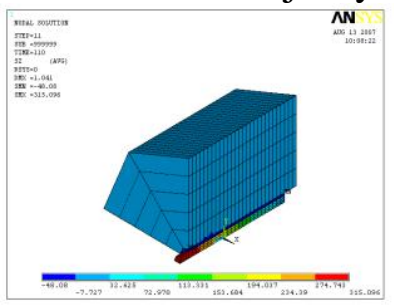

(a) FEA model

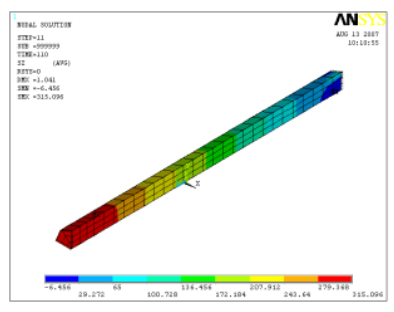

(b) Steel bar

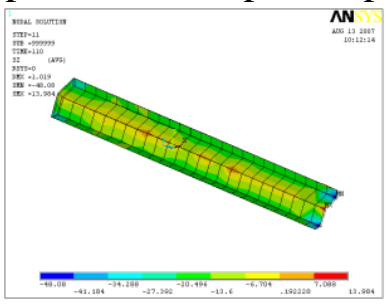

(c) Adhesive glue

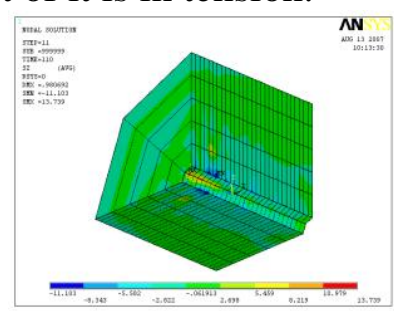

(d) Concrete block

Fig.6 Axial stress distribution

\section{Conclusions}

The three-phase bond-slip relationship, based on the results of pullout-test of adhesive steel bar in concrete, can be used in finite element analysis of the anchorage system. The bond-slip relationship reflects the effect of parameters, such as effective embedment length, drill diameter, characters of adhesive, etc., on bonding strength and slip of adhesive interface. The analytical result coincides with the experimental result, which proves that the analysis model is effective and the parameters are reliable.

The bonding behavior and failure model of adhesive steel in concrete can be studied efficiently by finite element analysis method. With the proposed FEA model, a lot of experiment could be saved and replaced by digital analysis. The bonding stress distribution on adhesive interface, the distribution and development of crack in concrete, the distribution of stress in steel, adhesive and concrete for different anchoring sample in whole loading process can be expressed in detail through digital computation.

\section{Acknowledgements}

This work was financially supported by National Natural Science Foundation of China (51178329).

\section{References}

[1] Jianrong Zhang, Lizhong Shi, Jin Wu, Xin Zhang and Mengwei Zhang: Experimental Research on the Behavior of Adhesive Reinforcement under Tensile Load, Structural Engineers [J]. Vol.20-5(2004), p.47-51, in Chinese.

[2] Jianrong Zhang, Lizhong Shi, Jianhua Yang, Jin Wu, Xin Zhang and Mengwei Zhang: Experimental Research on the Behavior of Chemical Adhesive Steel Bar in Concrete under Tensile Load [J]. Building Structure, Vol.36-3(2006), p.17-21, in Chinese.

[3] Ronald A. Cook, Member, ASCE. Behavior of Chemically Bonded Anchors [J]. Journal of Structural Engineering, September, 1993, Vol.119(9), 2744-2762.

[4] Technical Specification for Post-installed Fastenings in Concrete Structures (JGJ145-2013) [S], Industry Standard of the People Republic of China, in Chinese.

[5] Michael McCay, Ronald A. Cook, Kailash Krishnamurthy. Pullout Simulation of Post-installed Chemically Bonded Anchors [J]. Journal of Structural Engineering, September, 1996, Vol.122(9), 1016-1024. 